\title{
THE ROLE OF CA 19-9 AND PLATELET LYMPHOCYTE RATIO (PLR) IN THE DIAGNOSIS OF PANCREATIC HEAD MASS
}

\author{
Nagesh N. S1, Yogesh Biradar ${ }^{2}$
}

1 Professor and HOD, Department of Surgical Gastroenterology, BMCRI, Bengaluru, Karnataka, India.

${ }^{2}$ Resident, Department of Surgical Gastroenterology, BMCRI, Bengaluru, Karnataka, India.

ABSTRACT
BACKGROUND
Laboratory analysis is of limited benefit in the diagnosis of pancreatic cancer. Several tumour markers are available that can help
in prognostication and diagnosis of pancreatic cancer. Carbohydrate antigen 19-9 (CA 19-9) is the traditionally accepted best
marker available. However, its usefulness has two significant limitations. First, it is not specific for pancreatic cancer because it can
be elevated in benign conditions, particularly those that cause obstructive jaundice. Secondly, its sensitivity is reduced by the fact
that patients who test negative for Lewis blood group antigens A and B are unable to synthesise CA 19-9 and therefore do not
express it in their serum. The role of new tumour marker Platelet Lymphocyte Ratio (PLR) has been defined recently in
prognostication of carcinoma pancreas. Role of PLR in diagnosing and its efficacy after combining it with CA 19-9 is not well
established. This study is being undertaken to assess the role of Platelet Lymphocyte Ratio (PLR) and CA 19-9 in determining
nature of pancreatic head masses.

The objective of the study is to assess the role of PLR and CA 19-9 in the diagnosis and management of pancreatic head mass; assess short-term (3 months) post-operative outcome in pancreatic head mass cases with high PLR ratio.

\section{MATERIALS AND METHODS}

It is a descriptive study. Patients suffering from neoplastic and inflammatory pancreatic head masses admitted in the Department of Surgical Gastroenterology and Liver Transplant in Bangalore Medical College and Research Institute, Bangalore, were taken for the study. This is a prospective study from October 2015 to November 2017. A total of 30 patients were included in the study. The sample size was taken for convenience during the study.

\section{RESULTS}

In this study, accuracy of CA $19-9$ (37 U/mL) in differentiating carcinoma from chronic pancreatitis is $85 \%$ and $87 \%$ respectively. The accuracy of PLR in differentiating carcinoma from chronic pancreatitis is $84 \%$ and $83 \%$ respectively. But the accuracy for combination of CA $19-9$ and PLR in differentiating carcinoma from chronic pancreatitis is $88 \%$ and $90 \%$ respectively.

\section{CONCLUSION}

The preoperative Platelet Lymphocyte Ratio (PLR) has been reported as a significant prognostic indicator in several digestive malignancies. Use of both CA 19-9 and PLR resulted in a significant improvement in the ability to identify those patients in pancreatic head malignancy in whom supplementary staging can be avoided safely. PLR, which has been used for prognostic purpose till now also has a diagnostic value. PLR is at least as good as CA 19-9 as diagnostic marker to differentiate between malignant and inflammatory head mass of pancreas. Combining CA 19-9 and PLR may increase the accuracy.

\section{KEY WORDS}

CA 19-9 - Carbohydrate Antigen 19-9, PLR - Platelet Lymphocyte Ratio.

HOW TO CITE THIS ARTICLE: Nagesh NS, Biradar Y. The role of CA 19-9 and platelet lymphocyte ratio (PLR) in the diagnosis of pancreatic head mass. J. Evolution Med. Dent. Sci. 2018;7(35):3918-3922, DOI: 10.14260/jemds/2018/876

\section{BACKGROUND}

Pancreatic head mass constitutes an important clinical entity in gastrointestinal surgical practice. In these most common being adenocarcinoma of the head of pancreas, which is followed by the inflammatory masses due to chronic pancreatitis.

Accurate diagnosis is of main importance, as therapeutic strategies range from observation to major surgery like Whipple's surgery (Pancreaticoduodenectomy).

'Financial or Other Competing Interest': None.

Submission 21-06-2018, Peer Review 08-08-2018,

Acceptance 16-08-2018, Published 27-08-2018.

Corresponding Author:

Dr. Yogesh Biradar,

08-06-35, Biradar Hospital,

J. P. Nagar, Behind Government Medical College,

Bidar-585401, Karnataka, India.

E-mail: ybiradar01@gmail.com

DOI: $10.14260 /$ jemds $/ 2018 / 876$

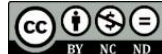

Several tumour markers are available that can help in prognostication and diagnosis of pancreatic cancer. Carbohydrate antigen 19-9 (CA 19-9) is the traditionally accepted best marker available. Ongoing research has produced a multitude of potential biomarkers for pancreatic cancer. The only biomarker that has recognised clinical utility is Carbohydrate antigen 19-9 (CA 19-9). However, its usefulness has two significant limitations. First, it is not specific for pancreatic cancer because it can be elevated in benign conditions, particularly those that cause obstructive jaundice. Second, its sensitivity is reduced by the fact that patients who test negative for Lewis blood group antigens $\mathrm{A}$ and B are unable to synthesise CA 19-9 and therefore do not express it in their serum. The percentage of pancreatic cancer patients who fall in this group has been reported to range from $10 \%$ to $34 \%$. Thus, CA $19-9$ best serve as a marker of treatment response and recurrence in patients who have a pathologic diagnosis of pancreatic cancer. 
Role of PLR in diagnosing and its efficacy after combining it with CA 19-9 is not well established. This study was undertaken to assess the role of Platelet Lymphocyte Ratio (PLR) and CA 19-9 in determining nature of pancreatic head masses. In this the short-term (3 months) post-operative outcome in pancreatic head mass cases with high PLR ratio is also assessed.

\section{Objectives}

\section{This study is undertaken to-}

1. To assess the role of PLR and CA 19-9 in the diagnosis and management of pancreatic head mass.

2. To assess short-term ( 3 months) post-operative outcome in pancreatic head mass cases with high PLR ratio.

\section{MATERIALS AND METHODS}

It is a descriptive study. The patients suffering from neoplastic and inflammatory pancreatic head masses admitted in the Department of Surgical Gastroenterology and Liver Transplant in Bangalore Medical College and Research Institute, Bangalore, were taken for the study.

This is a prospective study from October 2015 to November 2017.

A total of 30 patients were included in the study. The sample size was taken for convenience during the study.

All cases with impression of mass of pancreatic head on imaging indicated by the presence of abrupt focal change in gland thickness or thickness more than $3.5 \mathrm{~cm}$ of pancreatic head were included.

After obtaining clearance and approval from the Institutional Ethics Committee, the admitted patients at Surgical Gastroenterology ward of Bangalore Medical College which were diagnosed as pancreatic head mass fulfilling the criteria were enrolled in the study.

Demographics of admitted patients were noted. Relevant history such as jaundice, weight loss $>10 \%$ in the last $3-6$ months was considered significant), previous attack of pancreatitis and significant alcohol intake were noted. (Equivalent of $80 \mathrm{gm}$ or more of ethanol/ day for at least 5 years).

Patients admitted with a mass in the head of pancreas were subjected to these blood investigations as mentioned below-

- Complete blood count.

- Liver function tests.

- CA 19-9.

- PLR

\section{The following imaging studies were performed}

- Transabdominal ultrasonography.

- Doppler to see the status of the vessel where indicated.

- Contrast Enhanced Computed Tomography (CECT) abdomen (pancreas protocol CT), that is triphasic CT with arterial, pancreatic parenchymal and portal phase.

- $\quad$ MRI/ MRCP where indicated; for example, for diagnostic dilemma where CT cannot be done due to poor renal status or in condition like pregnancy.

- Upper gastrointestinal endoscopy- end/ side viewing scopy.

- Endoscopic Retrograde Cholangiopancreatography (ERCP) where indicated; for example, for diagnostic dilemma and where stenting was needed for palliation or cholangitis.

- Endoscopic Ultrasound (EUS) where indicated; for example, where diagnostic dilemma, about the nature of pancreatic head mass was present with other imaging techniques and EUS-Guided Fine Needle Aspiration Cytology (FNAC) could be taken.

- FNAC: Endoscopic Ultrasound-guided or CT-guided percutaneous for masses of indeterminate aetiology and metastatic tumours where chemotherapy would require pathologic confirmation.

All the patients were subjected to ultrasound and CECT (pancreas protocol) and upper GI endoscopy. Other tests were selectively used where indicated.

In short-term (3 months) postoperative follow-up, following criteria were taken into consideration:

POPF (Post-Operative Pancreatic Fistula) occurs with the leakage of amylase rich fluid from the transection margin of the gland and/ or from the pancreatic-enteric anastomosis. According to classification by the International Study Group of Pancreatic Surgery (ISGPS)-

1. Grade A fistulas are biochemical only and not clinically relevant.

2. Grade B require further evaluation and management such as antibiotics, nutritional support, octreotide and percutaneous drainage.

3. Grade $\mathrm{C}$ need surgical exploration in the setting of sepsis.

\section{SSI (Surgical Site Infection)}

- Superficial incisional, affecting the skin and subcutaneous tissue.

- Deep incisional, affecting the fascial and muscle layers.

- Organ or space infection, which involves any part of the anatomy other than the incision that is opened or manipulated during the surgical procedure.

\section{Improvement}

Means symptomatic improvement when compared to preoperative status in terms of pain, vomiting, jaundice, loss of weight and loss of appetite.

\section{Deterioration}

Means symptomatically becoming bad when compared to preoperative status in terms of pain, vomiting, jaundice, loss of weight and loss of appetite.

\section{Statistical Analysis \\ A descriptive statistical analysis was carried out in the present study. Student t-test (two-tailed, independent) was used to find the significance of study parameters on continuous scale between two groups. Inter-group analysis Chi-square/ Fisher exact test was used to find the significance of the study parameters on categorical scale between two or more groups. Mann-Whitney test was used.}

\section{RESULTS}

A total of 30 patients were included in this study. $46.7 \%$ of patients belong to 46 to 60 years of age group. Only $3.3 \%$ of patients belong to 76 to 90 years of age group. Mean age group in this study is 51.13 years. 
In this study, $76.7 \%$ of patients were males and $23.3 \%$ patients were females and $53.3 \%$ of patients were alcoholic and remaining were non-alcoholic.

$66.7 \%$ of patients had history of weight loss, whereas the remaining had no similar history. In this study, 25 patients had malignant disease and 5 patients had chronic pancreatitis (Benign). Amongst the malignant disease 21 had adenocarcinoma, 3 had metastatic disease and 1 had NET.

In the study, $43.3 \%$ of patients did not undergo surgery. Out of 17 patients who had undergone surgery, 07 patients developed Post-Operative Pancreatic Fistula (POPF), 09 patients had Surgical Site Infection (SSI), 12 patients improved, 3 patient's condition deteriorated, and 2 patients died during the short period of follow-up.

Different parameters were compared in malignant and benign patients. Following observations were made:

An average Lymphocyte count of 1299.24 / cu.mm was seen in patients with carcinoma, whereas it was 2781 /cu.mm in pancreatitis patients.

An average platelet count of $318812 /$ cu.mm was seen in patients with carcinoma, whereas it was $260800 /$ cu.mm in pancreatitis patients.

An average CA $19-9$ of $261.98 \mathrm{U} / \mathrm{L}$ was seen in patients with carcinoma, whereas it was $20.32 \mathrm{U} / \mathrm{L}$ in pancreatitis patients.

An average PLR of 261.98 was seen in patients with carcinoma, whereas it was 104.17 in pancreatitis patients.

Marked thrombocytosis ( $>4 \mathrm{~L}$ ) was seen in 6 patients. Of them, 5 were patients with carcinoma and 1 was with pancreatitis.

Marked lymphocytopenia ( $<1000 /$ cu.mm) was seen in 8 patients. All of them were patients with carcinoma.

PLR (when cut-off taken as 150) was taken as a marker to differentiate cancer from chronic pancreatitis.

CA 19-9 was seen in this study at various cut-off levels to see its effect.

Combination of CA 19-9 and PLR was also evaluated in this study.

\begin{tabular}{|c|c|c|c|c|}
\hline \multirow{2}{*}{ Parameters } & \multicolumn{2}{|c|}{ Carcinoma } & \multirow{2}{*}{ T value } & P \\
\cline { 2 - 5 } & Yes & No & 0.027 & $0.911^{* *}$ \\
\hline $\begin{array}{c}\text { Bilirubin } \\
\text { mg/dL }\end{array}$ & $2.7(10.3)$ & $8.04(9.9)$ & 0.227 & $0.026^{*}$ \\
\hline $\begin{array}{c}\text { Lymphocytes } \\
\text { Count/cumm }\end{array}$ & $\begin{array}{c}1299.24 \pm \\
591.55\end{array}$ & $\begin{array}{c}2781.00 \pm \\
992.2\end{array}$ & $3.263^{*}$ \\
\hline $\begin{array}{c}\text { Platelets } \\
\text { Lakhs/cumm }\end{array}$ & $\begin{array}{c}318812.00 \pm \\
111137.27\end{array}$ & $\begin{array}{c}260800.00 \pm \\
106797.47\end{array}$ & 1.071 & $0.293^{*}$ \\
\hline $\begin{array}{c}\text { Neutrophil } \\
\text { count/cumm }\end{array}$ & $\begin{array}{c}9369.16 \pm \\
4791.09\end{array}$ & $\begin{array}{c}8781.80 \pm \\
1994.42\end{array}$ & 0.449 & $0.660^{*}$ \\
\hline CA 19-9 U/L & $\begin{array}{c}261.98 \pm \\
329.16\end{array}$ & $\begin{array}{c}20.32 \pm \\
9.43\end{array}$ & 3.663 & $0.001^{*}$ \\
\hline PLR & $\begin{array}{c}281.97 \pm \\
201.03\end{array}$ & $\begin{array}{c}104.17 \pm \\
53.68\end{array}$ & 1.939 & $0.063^{*}$ \\
\hline
\end{tabular}

Table 1. Comparison of different parameters by Carcinoma

*Independent t-test and data presented Mean \pm Standard deviation.

** Mann-Whitney U-test used and data presented in Median (Interquartile range).

\section{DISCUSSION}

An inflammatory mass due to chronic pancreatitis and masses due to pancreatic carcinoma are the two most common causes of mass formation of head of pancreas.

Important haematological parameters that were assessed in the two groups in this study were platelet and lymphocyte counts.

Taking only the absolute lymphocyte count and platelet count into consideration yielded poor results as tumour markers. Even marked lymphocytopenia and marked thrombocytosis cannot be used as tumour markers because of poor accuracy. Combining both the haematological indices as Platelet Lymphocyte Ratio (PLR) reflects a novel marker.(1)

In a study by Senthilkumar Perumal et al in 2013 multivariate analysis of the risk factors have shown that CA 19.9 as the only significant risk factor predicting malignancy in patients with Chronic Calcific Pancreatitis (CCP) and head mass. CA 19-9 is useful in establishing the diagnosis of pancreatic cancer(2) with a sensitivity of $80-85 \%$ and specificity of $85-90 \%{ }^{(3,4)}$

In a study by Shirai Y in 2015,(5) a pre-operative PLR of $\geq 150$ was a significant and independent risk factors for cancer recurrence and poor survival respectively.

In a study by Richard A Smith in 2009,(6) the P/L ratio is a superior prognostic marker when compared with either individual parameter or neutrophil-lymphocyte ratio.

Initially evaluated by Smith et al in 2008 ,(7) this marker is being used for prognostic purpose in the cancer of pancreas. In their study to evaluate whether the preoperative PLR improve the predictive value of CA 19-9 levels in stratifying a patient group with suspected periampullary malignancy who do not require staging laparoscopy.

In a study by MMS Bedi et al in 2009,(8) it is shown that raising the threshold level of CA $19-9$ to $300 \mathrm{u} / \mathrm{mL}$ increases its predictive value as a positive test to $100 \%$.

In the present study, PLR (when cut-off was taken as 150) was taken as the marker to differentiate cancer from chronic pancreatitis. To the best of our knowledge, this is one of the very few studies that is using this parameter for this purpose.

The carbohydrate antigen 19-9 (CA 19-9) has been a useful marker, both in diagnosis as well as follow-up of pancreatic cancer. However, CA 19-9 is also elevated in inflammatory lesions of the pancreas.

Similar results were seen in the present study. It was observed that on increasing the cut-off value, NPV falls and sensitivity decreases, and the overall accuracy also comes down.

In our study, accuracy of CA $19-9(37 \mathrm{U} / \mathrm{mL})$ in differentiating carcinoma from chronic pancreatitis is $84 \%$ and $87 \%$ respectively. The accuracy of PLR in differentiating Carcinoma from Chronic pancreatitis is $84 \%$ and $83 \%$ respectively. But the accuracy for combination of CA 19-9 and PLR in differentiating Carcinoma from Chronic pancreatitis is $88 \%$ and $90 \%$ respectively.

In a similar study by Ashwin Rammohan et al in 2015,(9) accuracy of CA 19-9 in differentiating Carcinoma from Chronic pancreatitis is $85.45 \%$ and $89.16 \%$ respectively. Accuracy of PLR in differentiating Carcinoma from Chronic pancreatitis is $81.82 \%$ and $85.54 \%$ respectively. But the accuracy for combination of CA 19-9 and PLR in differentiating Carcinoma from Chronic pancreatitis is $94.55 \%$ and $96.39 \%$ respectively.(10,11) 
In another similar study by Ripan $\mathrm{K}$ Miglani et al in 2013,(1) PLR (when cut-off taken as 150) was taken as the marker to differentiate cancer from chronic pancreatitis. Sensitivity, specificity, PPV, NPV and accuracy were $63.64 \%$, $73.91 \%, 70.00 \%, 60.00 \%$ and $68.89 \%$.

CA 19-9 was seen in a study at various cut-off levels to see its effect. Sensitivity, specificity, PPV, NPV and accuracy at a cut-off $37 \mathrm{U} / \mathrm{mL}$ was $59.09 \%, 78.26 \%, 72.22 \%, 66.67 \%$ and $68.89 \%(\mathrm{p}=0.011)$. $^{(12)}$

Combination of CA 19-9 and PLR was evaluated in one study.(13) With this combination sensitivity, specificity, PPV, NPV and accuracy of $81.82 \%, 60.87 \%, 66.67 \%, 77.78 \%$ and $71.11 \%$, respectively $(\mathrm{p}=0.003)$.

With this study it was shown that combining PLR and CA19-9 gave the most accurate result in diagnosing carcinoma of pancreas.

In our study, results showed that there is a trend towards increased insensitivity and increase in overall accuracy when PLR is combined with CA 19-9 as compared with either tumour marker alone. When PLR alone was compared with CA 19-9, it showed similar accuracy to CA $19-9$ as a tumour marker.

Hence, there is a case for combining CA 19-9 with PLR to improve sensitivity for diagnosing cancer of pancreas. Also, PLR is a good replacement for CA 19-9, which is a costly tumour marker.

Such combination has not been tried to diagnose cancer of the pancreas. Hence, it was shown in the study that combining PLR and CA 19-9 gave the most accurate result in diagnosing carcinoma of pancreas, although this should be confirmed through larger studies.

Combining PLR and CA 19-9 also has a potential of diagnosing the development of carcinoma in the setting of pre-existing chronic pancreatitis and differentiating malignant mass from inflammatory mass in such cases. This is because altered ratio of platelets to lymphocytes is due to the factors that are released only in malignancy and not in benign condition. This needs to be confirmed in welldesigned larger studies.

Out of 17 patients who had undergone surgery during short period (3 months) of follow-up, 07 patients developed Post-Operative Pancreatic Fistula (POPF). Out of these, 06 were Grade A type and only 01 was of Grade B type.

09 patients had Surgical Site Infection (SSI). Out of these, 05 were superficial incision type and 04 were deep incision type. 12 patients improved symptomatically.

All the 12 patients got relieved of pain over this period. In 8 patients who had history of loss of appetite and loss of weight, they showed improvement as there was improvement in their appetite and also gained weight over this period.

In 4 patients who had jaundice and vomiting prior to surgery showed improvement over this period of postoperative follow-up as jaundice disappeared along with vomiting. Over this period of follow-up in 3 patients, condition deteriorated. In 2 patients, there was persisting pain with loss of appetite.

In one patient there was loss of appetite, loss of weight with persisting occasional vomiting. Two patients died during this short period of follow-up who had metastatic adenocarcinoma. These findings are in comparison with other studies.(14,15)

\section{CONCLUSION}

The preoperative Platelet Lymphocyte Ratio (PLR) has been reported as a significant prognostic indicator in several digestive malignancies. Use of both CA 19-9 and PLR resulted in a significant improvement in the ability to identify those patients in pancreatic head malignancy in whom supplementary staging can be avoided safely.

In this study, we were able to show that new tumour marker, that is PLR which has been used for prognostic purpose till now also has a diagnostic value.

PLR is at least as good as CA 19-9 as diagnostic marker to differentiate between malignant and inflammatory head mass of pancreas. Combining CA 19-9 and PLR may increase the accuracy.

Role of PLR may be more useful in detecting carcinoma that developed over chronic pancreatitis, as CA 19-9 is not very sensitive in these cases, but this aspect should be explored in further studies.

In the study, $43.3 \%$ of patients did not undergo surgery. Out of 17 patients who had undergone surgery, 07 patients developed Post-Operative Pancreatic Fistula (POPF). 09 patients had Surgical Site Infection (SSI). 12 patients improved, 3 patients' condition deteriorated, and 2 patients died during the short period of follow-up.

All the results of this study should be confirmed by additional studies involving larger sample size.

Masses in and around pancreas constitute an important clinical entity in gastrointestinal surgical practice. Most common being adenocarcinoma of head of pancreas followed by inflammatory masses due to chronic pancreatitis. Accurate diagnosis is of central importance, as therapeutic strategies range from observation to complete surgical removal including total pancreatectomy.

Several tumour markers are available, which could help in prognostication and diagnosis of carcinoma pancreas. Carbohydrate antigen 19-9 (CA 19-9) is traditionally accepted best marker available. The role of new tumour marker Platelet Lymphocyte Ratio (PLR) has been defined recently in prognostication of carcinoma pancreas. Role of PLR in diagnosing and its efficacy after combining it with CA 19-9 is not known.

The aim of study was to assess the demographics of neoplastic and inflammatory pancreatic head masses in our department. To assess the role of CA 19-9 and Platelet Lymphocyte Ratio (PLR) in determining nature of pancreatic head mass.

Data consisted of 30 patients. 05 patients were having head mass due to chronic pancreatitis and 25 because of neoplastic lesions. Demographics in terms of age, sex, previous pain episodes, presence of jaundice and history of alcohol intake were compared in both groups. Also, tumour markers CA 19-9 and PLR individually and in combination were compared in both groups.

Cancer pancreas significantly $(p<0.001)$ occurring in older age group was associated with jaundice. Accuracy in diagnosis of cancer pancreas was $87 \%$ with CA $19-9$ and $83 \%$ with PLR, whereas combining CA 19-9 with PLR showed increased sensitivity (88\%) and accuracy (90\%) in diagnosing cancer pancreas. Other combinations showed no advantage. 
PLR is at least as good as CA 19-9 as a diagnostic marker to differentiate between malignant and inflammatory head mass of pancreas.

\section{REFERENCES}

[1] Miglani RK, Bhateja N, Bhat RS, et al. Diagnostic role of Platelet Lymphocyte Ratio (PLR) in pancreatic head masses. Indian J Surg 2013;75(1):4-9.

[2] Minghini A, Weireter LJ Jr, Perry RR. Specificity of elevated CA 19-9 levels in chronic pancreatitis. Surgery 1998;124(1):103-5.

[3] Steinberg W. The clinical utility of the CA 19-9 tumorassociated antigen. Am J Gastroenterol 1990;85(4):350-5.

[4] Paganuzzi M, Onetto M, Marroni P, et al. CA 19-9 and CA 50 in benign and malignant pancreatic and biliary diseases. Cancer 1988;61(10):2100-8.

[5] Shirai Y, Shiba H, Sakamoto T, et al. Preoperative platelet to lymphocyte ratio predicts outcome of patients with pancreatic ductal adenocarcinoma after pancreatic resection. Surgery 2015;158(2):360-5.

[6] Smith RA, Bosonnet L, Raraty M, et al. Preoperative platelet-lymphocyte ratio is an independent significant prognostic marker in resected pancreatic ductal adenocarcinoma. The American Journal of Surgery 2009;197(4):466-72.

[7] Smith RA, Bosonnet L, Ghaneh P, et al. The plateletlymphocyte ratio improves the predictive value of serum CA 19-9 levels in determining patient selection for staging laparoscopy in suspected periampullary cancer. Surgery 2008;143(5):658-66.

[8] Bedi MM, Gandhi MD, Jacob G, et al. CA 19-9 to differentiate benign and malignant masses in chronic pancreatitis: is there any benefit? Indian J Gastroenterol 2009;28(1):24-7.
[9] Rammohan A, Cherukuri SD, Palaniappan R, et al. Preoperative platelet-lymphocyte ratio augments CA $19-9$ as a predictor of malignancy in chronic calcific pancreatitis. World J Surg 2015;39(9):2323-8.

[10] Falconi M, Casetti L, Salvia R, et al. Pancreatic head mass. How can we treat it? Chronic pancreatitis: surgical treatment. Journal of the Pancreas 2000;1(3):154-61.

[11] Bassi C, Falconi M, Caldiron E, et al. To what extent is surgery superior to endoscopic therapy in the management of chronic pancreatitis? Ital J Gastroenterol Hepatol 1998;30:571-9.

[12] Kozuscheck W, Reith HB, Waleczek H, et al. A comparison of long term results of the standard Whipple procedure and the pylorus preserving pancreaticoduodenectomy. J Am Coll Surg 1994;178(5):443-53.

[13] Di Carlo V, Zerbi A, Balzano G, et al. Pylorus-preserving pancreaticoduodenectomy versus conventional Whipple operation. World J Surg 1999;23(9):920-5.

[14] Jzbicki JR, Bloechle C, Knoefel WT, et al. Duodenum preserving resection of the head of the pancreas in chronic pancreatitis. A prospective, randomized trial. Ann Surg 1995;221(4):350-8.

[15] Saraee A, Vahedian-Ardakani J, Saraee E, et al. Whipple procedure: a review of a 7-year clinical experience in a referral center for hepatobiliary and pancreas diseases. World Journal of Surgical Oncology 2015;13:98. 